\title{
Research on Target Detection Algorithm of Radar and Visible Image Fusion Based on Wavelet Transform
}

\author{
Dahui LI*, Qi FAN, Jianzhao CUI, Dehai HUANG, Jinku HAN
}

\begin{abstract}
The target detection rate of unmanned surface vehicle is low because of waves, fog, background clutter and other environmental factors on the interference. Therefore, the paper studies the target detection algorithm of radar and visible image fusion based on wavelet transform. The visible image is preprocessed to ensure the detection effect. The multi-scale fractal model is used to extract the target features, and the difference between the fractal features of the target and the background is used to detect the target. The radar image is denoised by a combination of median filtering and wavelet transform. The processed visible light and radar image are fused with wavelet transform strategy. The coefficients of the low frequency sub-band are processed by the average fusion strategy. The coefficients of the high frequency sub-band are processed using a strategy with a higher absolute value. The standard deviation, the spatial frequency and the contrast resolution of the image fusion result are compared. The simulation results show that the processed image is better than the unprocessed image after the fusion.
\end{abstract}

Keywords: image fusion; target detection; wavelet transform

\section{INTRODUCTION}

Unmanned surface vehicles detect targets on the sea surface is the hotspot in recent years. The main imaging methods for the detection of the target are visible, infrared, radar and so on. Visible imaging is mainly used in the light of a better environment. Radars can effectively detect medium-to-long range targets. But there is a blind spot to detect short-range targets. Therefore, this paper proposes that the target image detected by visible and radar images should be fused to further detect the target.

In the researches of targets recognition based on visible, literature [1] proposed an image enhancement method that can clear Marine image. In the study of visible on the sea target recognition, literature [2] proposed guided filtering technique, the method retains the edge features of the image for image feature extraction and detection.

The millimeter wave combines the performance of microwave guidance and photoelectric guidance due to the wavelength of millimeter wave being between centimeter wave and light wave. At the same time millimeter wave radar on the rain, fog, smoke penetrating ability, has the advantage of all-weather, all-day [3]. Synthetic Aperture Radar (SAR) is a microwave imaging radar system with high resolution. Imaging mechanism and other factors cause the azimuth ambiguity noise appearing in SAR imaging. There will be false targets appearing on the image when the noise is in severe cases. The reason of false target appearance is analyzed, specific location decoy that appeared in SAR images is deduced [4]. The target detection algorithm of TSAR image has two parameters with constant false alarm rate (CFAR). In view of the sea micro target echo signal having the characteristics of sparseness, the sparse signal processing has been applied in radar targets detection, and detection performance is better than the traditional method [5]. On the basis of morphological analysis, literature [6] proposed a target detection algorithm which made full use of the morphological differences between sea surface echoes and micro-doppler signals and completed the extraction and detection of micro-motion signals. The moving radar target detection method based on high-resolution sparse representation is proposed in document [7]. So literature [8] firstly established the framework of Short-Time sparse Time-Frequency Distribution (ST-TFD). So Short-Time Sparse Fourier Transform and Short-Time Sparse Fractional Fourier Transform based radar moving target detection methods are proposed.

The development of image fusion technology plays a crucial role in improving the stability of battlefield control and command system. Aiming at the disadvantages of easy-to-loss target information and low contrast images of the fusion images of SAR and visible images in multi-scale fusion algorithm, a fast fusion algorithm of SAR and optical images based on improved $l_{1}$ norm and sparse representation is proposed to retain target information of the source images effectively [9]. Literature [10] proposed a fusion algorithm for SAR and visible images in the nonsubsample wavelet transform domain with Hidden Markov Model. The low frequency factorsare fused by standard deviation. Meanwhile, Hidden Markov Tree is utilized to train high frequency factors. So the final image obtained the low-frequency image. Under the framework of transform fusion, genetic algorithm may search the weighting coefficient of low-frequency, realize the highfrequency coefficient of SAR [11]. Literature [12] got fusion image by Non-sub-sampled Contourlet Transform (NSCT) to fuse the important objective information of SAR image into visible image, and retain detail information as much as possible using mathematical morphology and multi-scale space theory to extract the detail features of the source image, the fusion image with enhanced detail features. For the registration problem of SAR images, the visible image is used as the reference image. By fusing the region and characteristic attributes of the image, an automatic and robust registration method using classified template for the unlimited-length SAR images with templates is proposed [13]. Literature [14] used laser imaging radar to acquire data, giving data fusion method based on target features between laser radar image and passive optical image, and realizing three-dimensional fusion between laser image and optical image. The document [15], the laser radar image and visible light image are denoised and realized the fusion.

This paper carried out the image enhancement, threshold segmentation, multi-scale fractal processing and region growing for visible images. The passive millimeter 
wave radar image is denoised by combining wavelet transform and median filtering. The low frequency and the high frequency sub-bands of the processed visible image and the processed radar image are processed by using the wavelet transform fusion strategy, so that the detection and identification of the target are realized.

\section{THE TWODIMENSIONAL WAVELET TRANSFORM}

The twodimensional wavelet transform plays an important role in wavelet analysis. In the applications of image decomposition, compression, edge extraction and image filtering, the wavelet is all the twodimensional wavelet, so the research and construction of the twodimensional wavelet analysis is very important. In this paper, multi-scale fractal feature extraction and image fusion are performed using the twodimensional wavelet.

Multi-resolution analysis in $L^{2}(R)$ space refers to the closed subspace sequence $\left\{V_{j}\right\} j \in \mathrm{Z}$ which satisfies monotonicity, approximation, flexibility, translation invariance, and existence of Reisz basis. Let $f_{j+1}(x, y) \in$ $L^{2}\left(R^{2}\right), V_{j}^{2}(j \in Z)$ be the separable multi-resolution analysis of $L^{2}\left(R^{2}\right)$. And let $\phi(x, y)=\phi(x) \phi(y)$ be the corresponding two dimensional scaling function. $\psi(x)$ is one dimension orthonormal wavelet corresponding to scaling function. Defining three "two dimensional wavelets" is (1):

$$
\left\{\begin{array}{l}
\psi^{H}(x, y)=\varphi(x) \psi(y) \\
\psi^{V}(x, y)=\varphi(x) \psi(y) \\
\psi^{D}(x, y)=\varphi(x) \psi(y)
\end{array}\right.
$$
(2),

They are standard orthogonal bases in $L^{2}\left(R^{2}\right)$, therefore

$$
\begin{aligned}
& f_{j+1}(x, y)=\sum_{k, m} c_{k, m}^{j+1} \phi_{j, k, m}=\sum_{k, m} c_{k, m}^{j} \phi_{j, k, m}+ \\
& +\sum_{k, m} d_{k, m}^{j, 1} \psi_{j, k, m}^{H}+\sum_{k, m} d_{k, m}^{j, 2} \psi_{j, k, m}^{V}+\sum_{k, m} d_{k, m}^{j, 3} \psi_{j, k, m}^{D}
\end{aligned}
$$

The decomposition algorithm is obtained by using the orthogonality of scaling function and wavelet function (3):

$$
\left\{\begin{aligned}
c_{k, m}^{j} & =\sum_{l, n} h_{l-2 k} h_{n-2 m} c_{l, n}^{j+1} \\
d_{k, m}^{j, 1} & =\sum_{l, n} h_{l-2 k} g_{n-2 m} c_{l, n}^{j+1} \\
d_{k, m}^{j, 2} & =\sum_{l, n} g_{l-2 k} h_{n-2 m} c_{l, n}^{j+1} \\
d_{k, m}^{j, 3} & =\sum_{l, n} g_{l-2 k} g_{n-2 m} c_{l, n}^{j+1}
\end{aligned}\right.
$$

Reconstruction algorithm as (4):

$$
\begin{aligned}
& c_{k, m}^{j+1}=\sum_{l, n} h_{k-2 l} h_{m-2 n} c_{l, n}^{j}+\sum_{l, n} h_{k-2 l} g_{m-2 n} d_{l, n}^{j, 1}+ \\
& +\sum_{l, n} g_{k-2 l} h_{m-2 n} d_{l, n}^{j, 2}+\sum_{l, n} g_{k-2 l} g_{m-2 n} d_{l, n}^{j, 3}
\end{aligned}
$$

The twodimensional wavelet decomposition and reconstruction algorithms are separable. The first row is one dimensional wavelet transform. On this basis, the one dimension wavelet transform is completed according to the column. From the filter point of view, the two dimension wavelet transform is the image data in two dimensions on the role of two times filtering, so has sets $\left[c A_{j}, c D_{j}^{H}, c D_{j}^{V}, c D_{j}^{D}\right]$, where $c A_{j}$ is the low pass filter of $j$-layer. The horizontal component $c D_{j}^{H}$ of the detail parameter in the $j$ layer is obtained by the transversal pass through the low pass filter and the longitudinal action high pass filter. The vertical component $c D_{j}^{V}$ of the detail parameter in the $j$ layer is obtained by the transverse pass through the high pass filter and the longitudinal action low pass filter. The diagonal component $c D_{j}^{D}$ of the detail parameter in the $j$ layer is obtained by the high pass filter in the two dimensions.

\section{DESIGN OF IMAGES FUSION ALGORITHM}

The target detection rate of unmanned surface vehicle is low because of waves, fog, background clutter and other environmental factors on the interference. Therefore, this paper studies the target detection algorithm of the visible images and the radar images fusion by unmanned surface vehicle in the sea surface. The target is detected according to the difference of the fractal features between the target and the background, and the multi-scale fractal model is used to extract the target features. The radar image is denoised by median filtering and wavelet transform. The processed visible light and radar image are fused with wavelet transform strategy. The coefficients of the low frequency sub-band are processed by the average fusion strategy. The coefficients of the high frequency sub-band are processed using a strategy with a higher absolute value. In this way, the detection of the target is realized.

\subsection{Processing of Visible Images}

The approach of processing visible images is shown in Fig. 1. The multi-scale fractal algorithm is used to detect the target in visible images. Multi-scale fractal target detection algorithms include the preprocessing, threshold segmentation, multi-scale fractal feature extraction, target determination and region growth. Its purpose completed the multi-target detection of optical images.

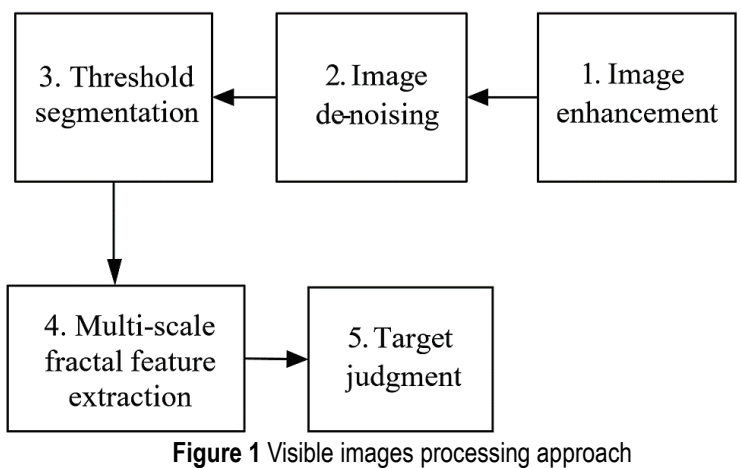

Figure 1 Visible images processing approach 
Let the input sequence be $\left\{x_{i}, I, I\right\}, I$ is a natural number set or subset, and the window length is $n$, then the filter output is (5).

$y_{i}=\operatorname{Med}\left\{x_{i}\right\}=\operatorname{Med}\left\{x_{i-u}, \ldots, x_{i}, \ldots, x_{i+u}\right\}$

where, $i \in I, u=(n-1) / 2$.

The threshold segmentation technique uses Otsu method. With the best threshold, the gray value of the image is divided into two parts, so that the variance between the two parts is the largest, and has the largest separation. The image is segmented to determine the number of potential target points.

Next, the image features are extracted by wavelet decomposition. The multi-scale fractal features are extracted by each center point with potential target. Due to the large difference between the multi-scale fractal features of the actual target points and other background subimages, the actual target points can be extracted from the image.

Finally, the target is judged. The natural background, such as wave and cloud, has fractal characteristics, while artificial targets such as ships do not have fractal characteristics. Therefore, the target is detected by using the difference of the fractal characteristics between the target and the background. Using fractal dimension threshold and multi-scale fractal feature threshold to judge target edge, ensure the stability of target detection and effectively eliminate the interference of ocean waves. Using continuous sub-images with high multi-scale fractal features, select a larger region as the target region. The centroid of the region is calculated, and the target region is obtained through the growth of the gray image region for region recognition.

\subsection{Processing of Radar Images}

The approach to processing radar images is shown in Fig. 2. Median filter can effectively suppress impulse noise, and wavelet threshold method can suppress the Gauss noise. For the image containing Gauss noise and impulse noise, and in order to remove the sea clutter and the same frequency interference, while retaining the complete target information, this paper uses the two methods. Firstly the original image is denoised by the median filter, secondly it is decomposed by two layers of wavelet. Thirdly the high frequency coefficients are quantized by threshold, and the two dimensional wavelet is reconstructed. Finally, the peak signal-to-noise ratio is used to objectively evaluate the effectiveness of this image preprocessing algorithm.
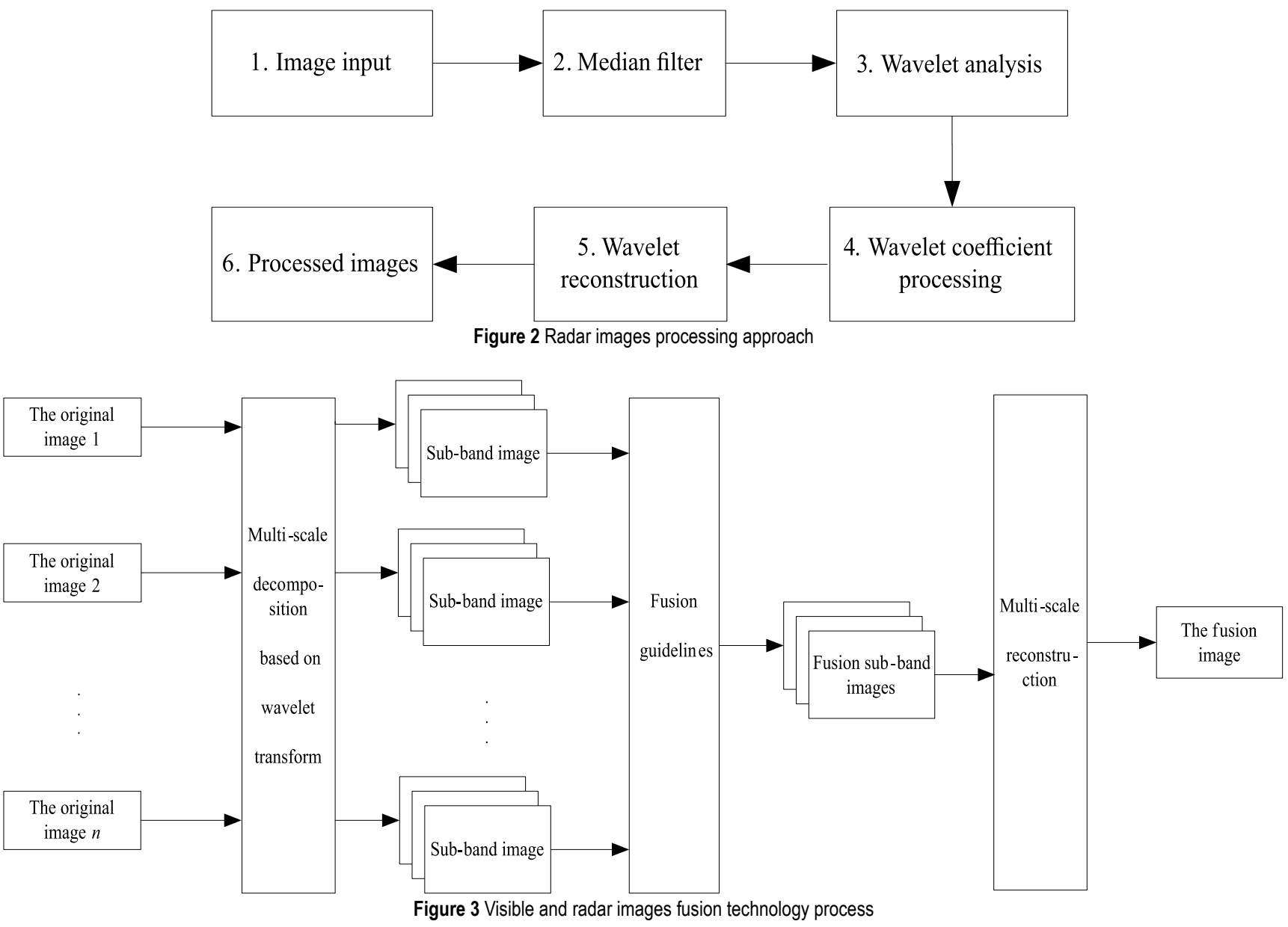

\subsection{Fusion Algorithm of Visible and Radar Images Based on Wavelet Transform}

The process of visible and radar images fusion technology is shown in Fig. 3. Using a variation approach to multi-scale of image signal decomposition methods, the images are projected onto their respective scales with high frequency details of the respective resolutions. The radar and visible image data are extracted and retained at multiscale and mufti-attribute. The image fusion algorithm 
based on wavelet transform is used to ensure the detection effect of the target image and improve the accuracy of image segmentation.

The image fusion method based on wavelet transform is to process each sub-band of each scale one by one. Wavelet transform can decompose the image data into multiple bands at different scales. After the wavelet transform, the coefficients of low frequency self-contained and high frequency self-contained are obtained.

Let $\boldsymbol{C}(X)$ represent the low frequency component coefficient matrix of wavelet decomposition of image $X$, and $p(m, n)$ represent the spatial location of wavelet coefficients. Therefore, $C_{J}(X, p)$ represents the value of the wavelet low frequency component coefficient matrix as the value of $(m, n)$ elements in the wavelet decomposition of the image $X$ in the $J$ layer. $\boldsymbol{G}(X, p)$ indicates the significant regional variance of $p$-centered. $\bar{u}(X, p)$ represents the matrix of low frequency coefficient of $X$, and is shown as (6).

$$
\boldsymbol{G}(X, p)=\sum_{q \in Q} w(q)|\boldsymbol{C}(X, p)-\bar{u}(X, p)|^{2}
$$

where, $w(q)$ represents the weight, the closer to $p$, the greater the weight. $M_{2}(p)$ is the low frequency coefficient of $A$ and $B$, the region variance matching degree of $p$ point is shown as (7).

$$
M_{2}(p)=\frac{2 \cdot \sum_{q \in Q} w(q)\left|\boldsymbol{C}_{\boldsymbol{J}}(A, p)-\bar{u}(A, p)\right|\left|\boldsymbol{C}_{\boldsymbol{J}}(B, p)-\bar{u}(B, p)\right|}{\boldsymbol{G}(A, p)+\boldsymbol{G}(B, p)}
$$

Set $T_{2}$ is the threshold of matching degree. When $M_{2}(p) \geq T_{2}$, the coefficients of the low frequency sub- band are processed by the average fusion strategy, such as Eqs. (8), (9) and (10).

$$
\begin{aligned}
& \boldsymbol{C}_{\boldsymbol{J}}(F, p)= \\
& =\left\{\begin{array}{l}
W_{\max } \boldsymbol{C}_{\boldsymbol{J}}(A, p)+W_{\min } \boldsymbol{C}_{\boldsymbol{J}}(B, p), \boldsymbol{C}_{\boldsymbol{J}}(A, p) \geq \boldsymbol{C}_{\boldsymbol{J}}(B, p) \\
W_{\min } \boldsymbol{C}_{\boldsymbol{J}}(A, p)+W_{\max } \boldsymbol{C}_{\boldsymbol{J}}(B, p), \boldsymbol{C}_{\boldsymbol{J}}(A, p)<\boldsymbol{C}_{\boldsymbol{J}}(B, p)
\end{array}\right. \\
& W_{\min }=0.5-0.5\left(\frac{1-M_{2}(p)}{1-T_{2}}\right) \\
& W_{\max }=1-W_{\min }
\end{aligned}
$$

The coefficients of the high frequency sub-band are processed using a strategy with a higher absolute value as (11)

$$
\boldsymbol{C}_{\boldsymbol{J}}(F, p)=\left\{\begin{array}{l}
\boldsymbol{C}_{\boldsymbol{J}}(A, p),\left|\boldsymbol{C}_{\boldsymbol{J}}(A, p)\right| \geq\left|\boldsymbol{C}_{\boldsymbol{J}}(B, p)\right| \\
\boldsymbol{C}_{\boldsymbol{J}}(B, p),\left|\boldsymbol{C}_{\boldsymbol{J}}(A, p)\right|<\left|\boldsymbol{C}_{\boldsymbol{J}}(B, p)\right|
\end{array}\right.
$$

The fused low frequency sub-band coefficients and high frequency sub-band coefficients are inversely reconstructed by wavelet. The image of the final data fusion result is reconstructed.

\section{COMPUTER SIMULATION}

This simulation is carried out under the MATLAB R2015a environment in the Windows 7 system. The visible light image and the radar image are respectively preprocessed, and the images are fused by a wavelet transformation fusion strategy to obtain a fusion image. Fig. 4 shows the overall simulation flow chart.

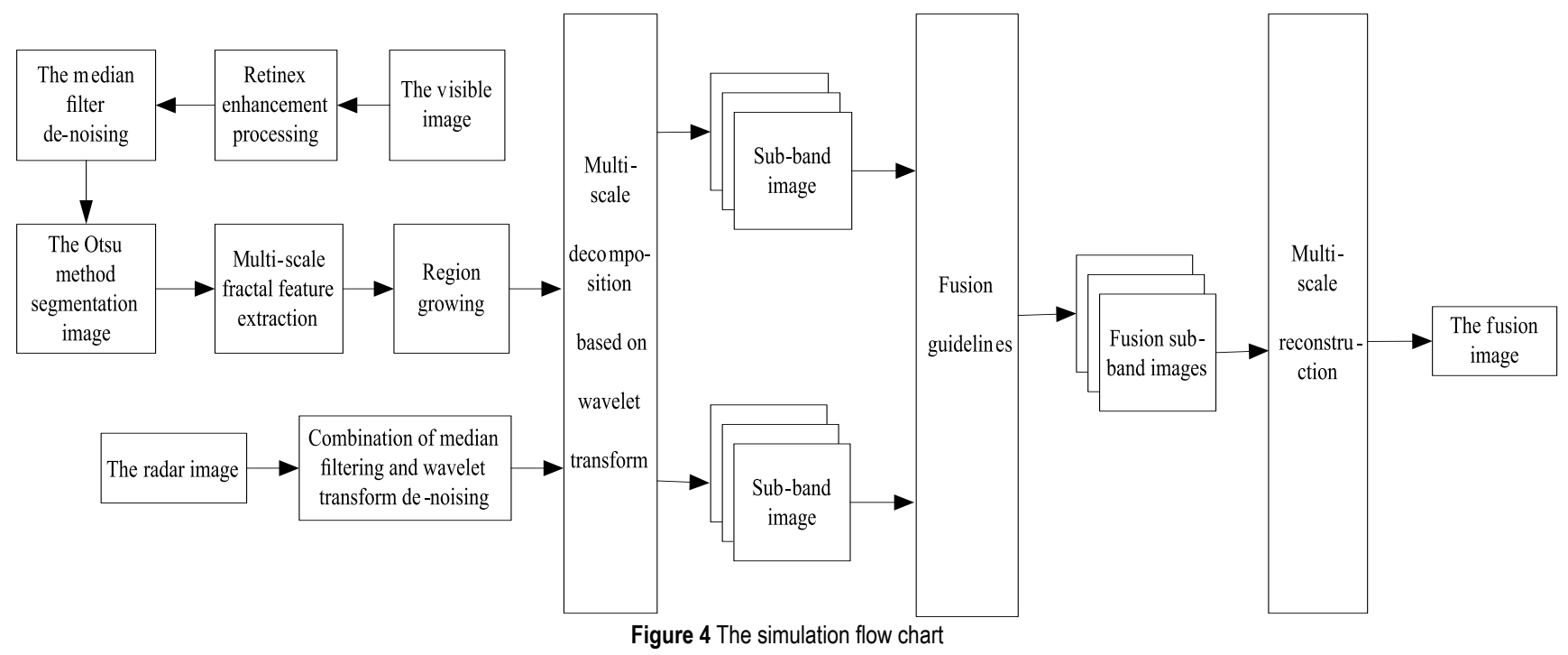

\subsection{To Complete the Visible Image of the Target Detection Simulation}

First of all, in order to eliminate the interference of the fog and noise for the original image, this paper adopted Retinex enhancement algorithm and median filter, as shown in Fig. 5a. Retinex enhancement processing is applied to three layers of $R, G$ and $B$ channels of RGB images respectively. Fig. $5 \mathrm{~b}$ is a Retinex enhanced image. Fig. 5c and Fig. 5d are images of before and after the treatment of the histogram. The Retinex image enhancement maintained the local image of the original image, and is displayed by histogram distribution. The 
result is smoother and the color characteristics are more natural. It has a good defogging effect.

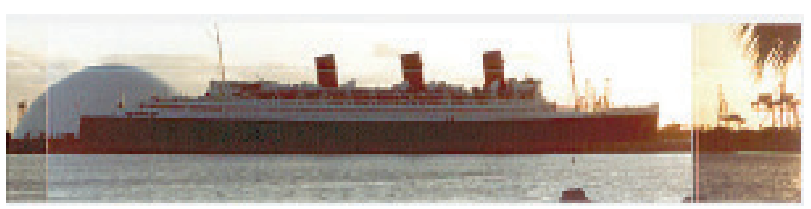

(a) The original image
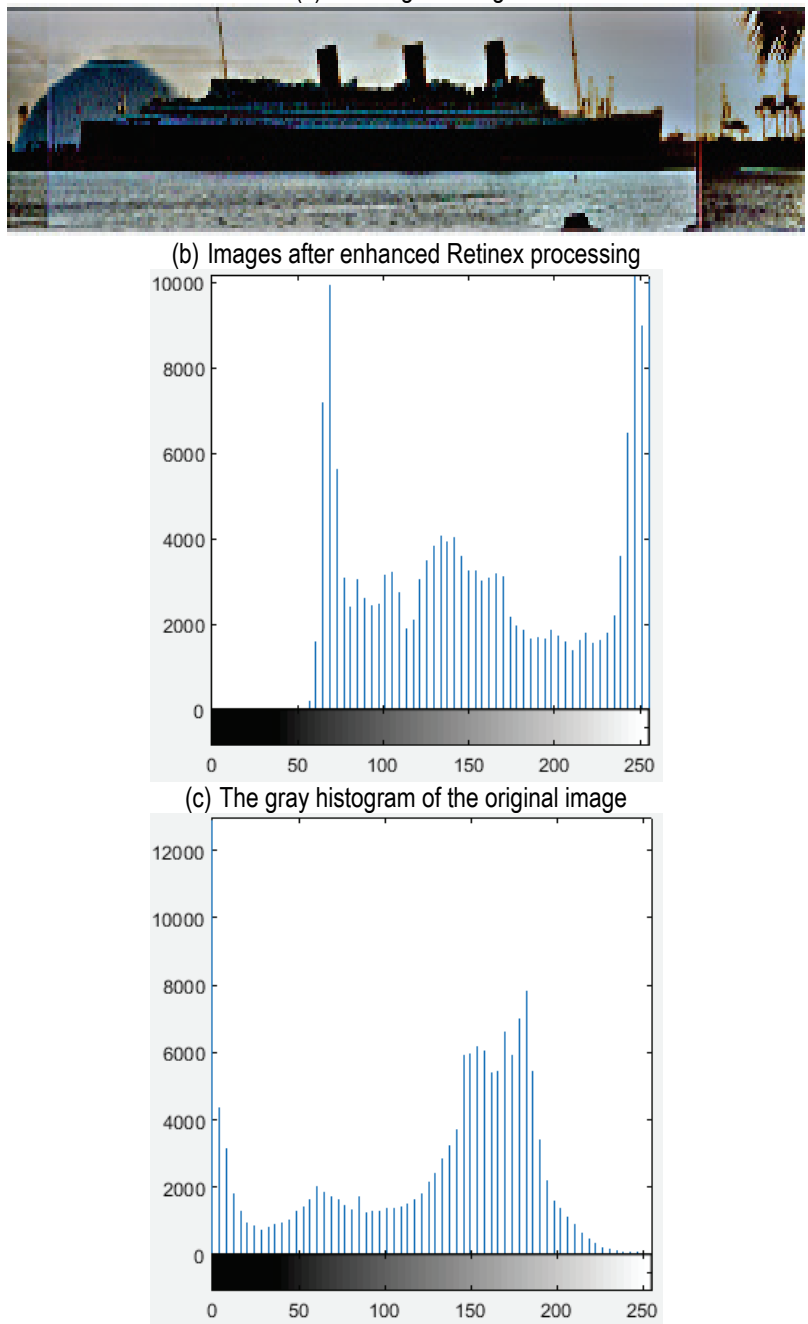

(d) The gray histogram after processing by Retinex Figure 5 The gray histogram

The image is denoised by median filter and compared to the two wavelet transform in Fig. 6.

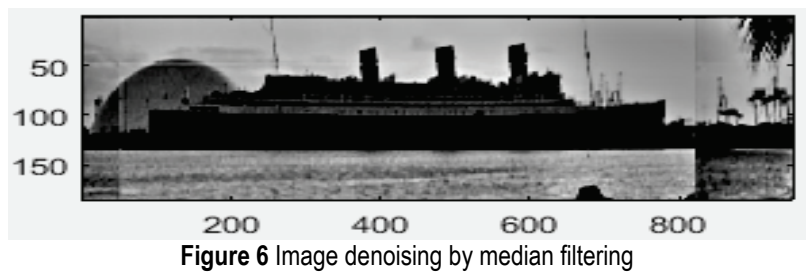

According to the threshold segmentation technique, the Otsu method is used to segment the target, as shown in Fig. 7. The optimal threshold of this graph is 90 by using the Otsu method. Finally, the multi-scale fractal features extract the image features. The actual target point is utilized to extract the multi-scale fractal feature of each potential target point, and is shown in Fig. 8.

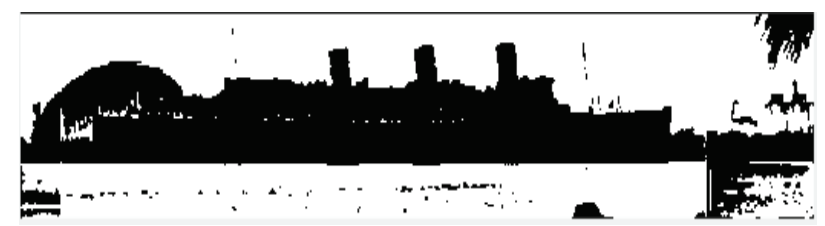

Figure 7 The threshold for image segmentation

Using continuous sub-images with high multi-scale fractal features, select a larger region as the target region. By grayscale images region growth to obtain the target region, the region recognition is carried out in Fig. 9.

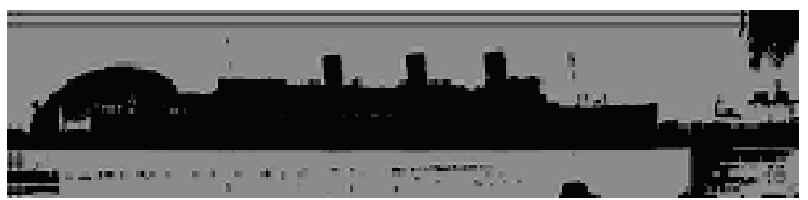

(a) The second layer approximation coefficients of image

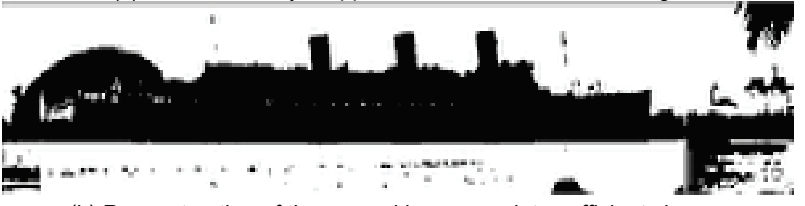

(b) Reconstruction of the second layer wavelet coefficients image

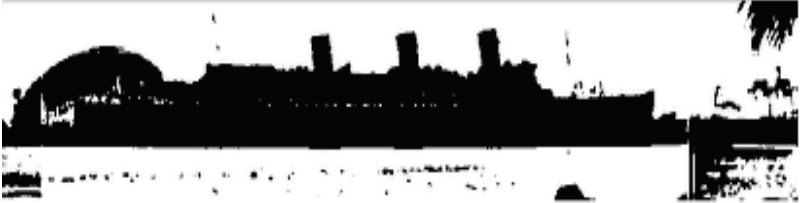

(c) The image with low frequency component synthesis approximation

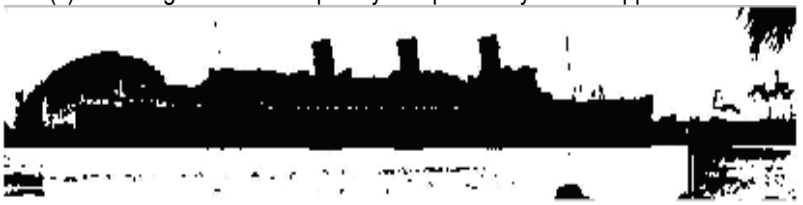

(d) The image with high frequency component synthesis approximation Figure 8 The multi-scale fractal features of image

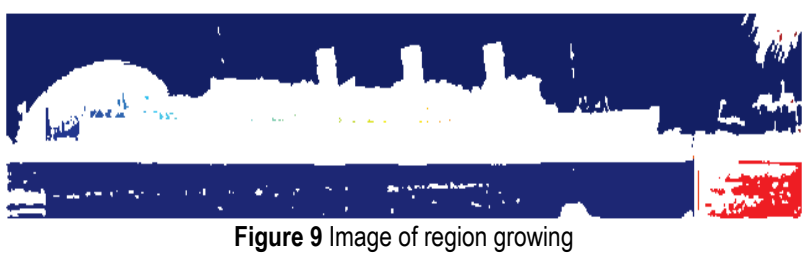

\subsection{To Complete the Radar Image of the Target Detection Simulation}

Fig. 10 is the original image of the radar. Fig. 11a shows the results of noise reduction with using median filtering. The radar image is processed by combining wavelet transform with median filtering and denoised by the global threshold function; the denoised image is obtained and shown in Fig. 11b and Fig. 11c.

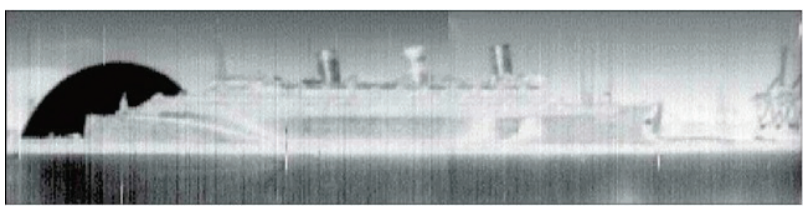

Figure 10 The original image of radar

Fig. 11c is based on Fig. 11b, the high frequency wavelet coefficients are processed again by using the 
threshold and getting the de-noised image. The Power Signal-to-Noise Ratio (PSNR) of the noise reduction algorithm is simulated as shown in Tab. 1.

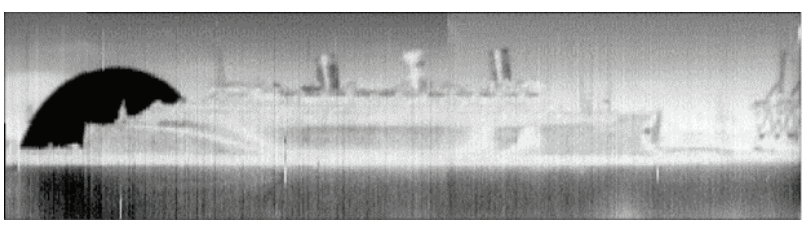

(a) The image by median filtering

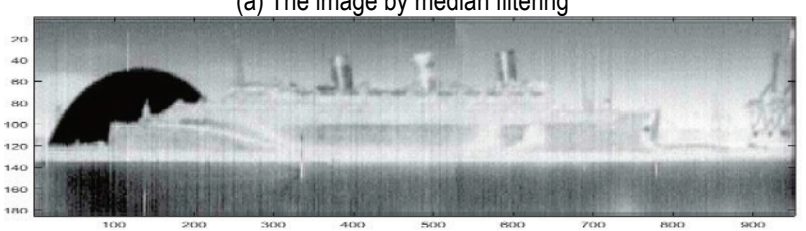

(b) The first image after combining

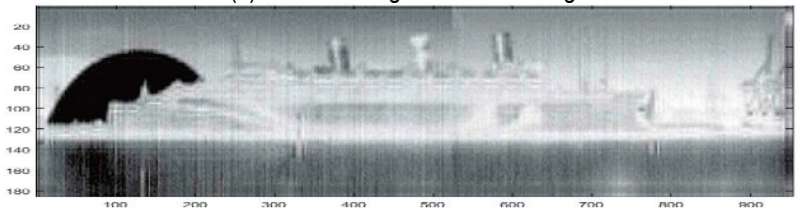

(c) The second image after combining

Figure 11The processing result of the radar image

Table 1 PSNR comparison table for radar image denoised

\begin{tabular}{|c|c|}
\hline Radar Image Denoised & PSNR / dB \\
\hline Fig. 10 & 6.5346 \\
\hline Fig. 11a & 6.6667 \\
\hline Fig. 11b & 7.4826 \\
\hline Fig. 11c & 7.4842 \\
\hline
\end{tabular}

$\operatorname{PSNR}(\mathrm{dB})$

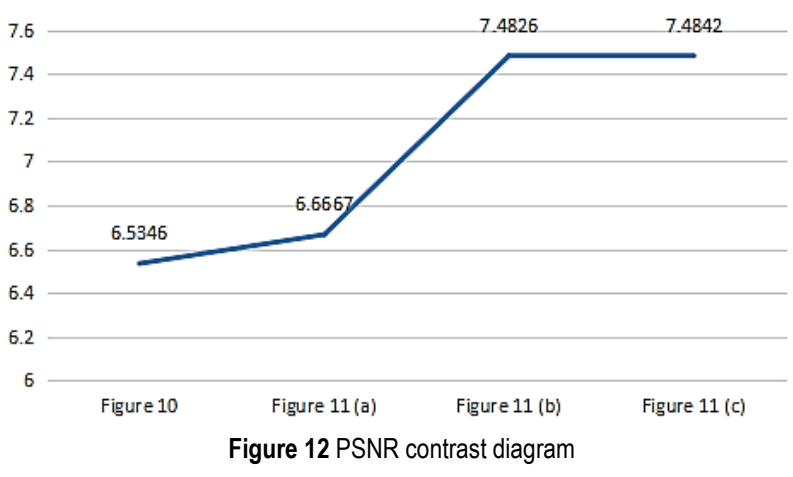

According to the data shown in Tab. 1 and Fig. 12, it can be seen that the PSNR of the global threshold filtering using the median filter and the wavelet transform in Fig. $11 \mathrm{c}$ is lower than that of the noise filtering using the median filter in Fig. 11a rather bigger. Therefore, the wavelet global threshold filtering method has good results of noise reduction.

\subsection{To Complete Target Detection Simulation of Visible and Radar Images Fusion Based on Wavelet Transform}

The image fusion method of wavelet transform may process each sub-band of each scale one by one. Wavelet transform can decompose the image data into multiple bands at different scales. After the wavelet transform, the coefficients of low frequency self-contained and high frequency self-contained are obtained. The coefficients of the low-frequency sub-bands are processed by the average fusion strategy. The high frequency sub-band coefficients are processed by the strategy of higher absolute value. The coefficients of the low frequency and high frequency subbands are inversely reconstructed by wavelet to obtain the fused image. Fig. 13 shows image processing according to this method. As shown in Fig. 13a, the grayscale visible image and the radar image are fused.

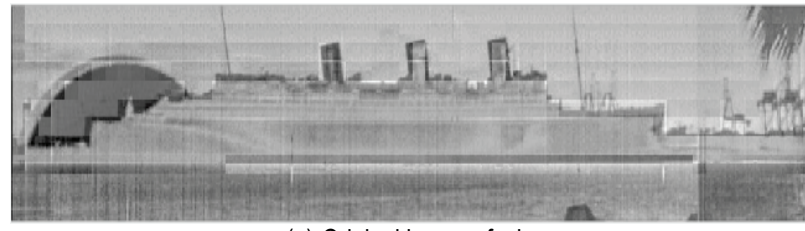

(a) Original images fusion

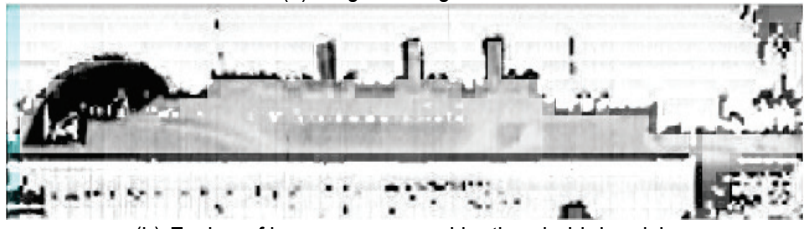

(b) Fusion of images processed by threshold denoising

Figure 13 Fused images

After wavelet decomposition and reconstruction, the visible image can be fused with the radar image. The coefficients of low frequency sub-bands and high frequency sub-bands are inversely reconstructed by wavelet transform to reconstruct the final data fusion result as shown in Fig. 13b. Figs. 14a and $14 \mathrm{~b}$ are the fused images obtained by performing different wavelet decomposition processing coefficients on the processed visible image and the radar image. Fig. 14a shows the image fusion for the average of the detail and the approximate signal. Fig. $14 \mathrm{~b}$ is the maximum of the approximate signal, the minimum of the detail signal for image fusion.

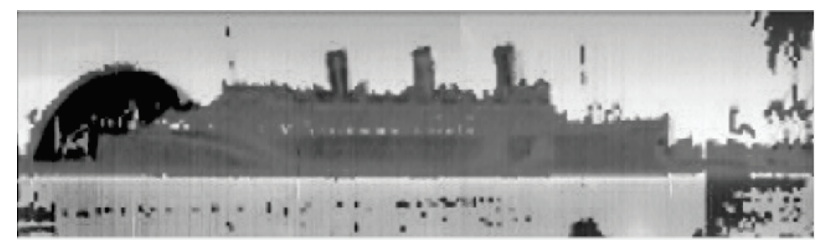

(a) Take the average of the detail and the approximate signal for fusion

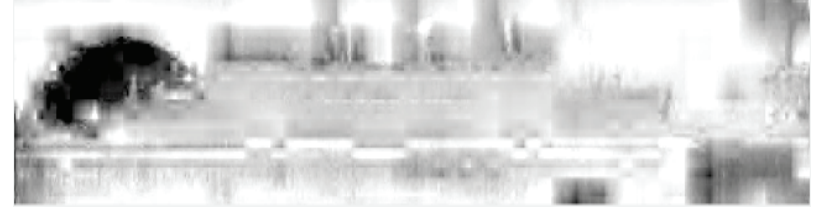

(b) Take the maximum of the approximate signal and the minimum of the detail signal for fusion

Figure 14 Fused images

The standard deviation, the spatial frequency, the sharpness and the information entropy factor are compared by the fusion result of the original image and the reconstructed image. The results are shown in Tab. 2 and Fig. 15. From the numerical analysis of performance indicators the results can be clearly seen. The performance indexes of the processed visible image and radar image fusion are better than those of the original image fusion in terms of standard deviation, spatial frequency and contrast resolution based on the fusion method of this paper. As the high frequency components are processed in the multiscale fractal feature extraction of visible images, the 
information entropy is slightly lower than the original image fusion. Moreover, the processed visible light image is fused with the radar image, and its performance indexes are obviously lower than the fusion method adopted in this article when adopting other fusion methods.

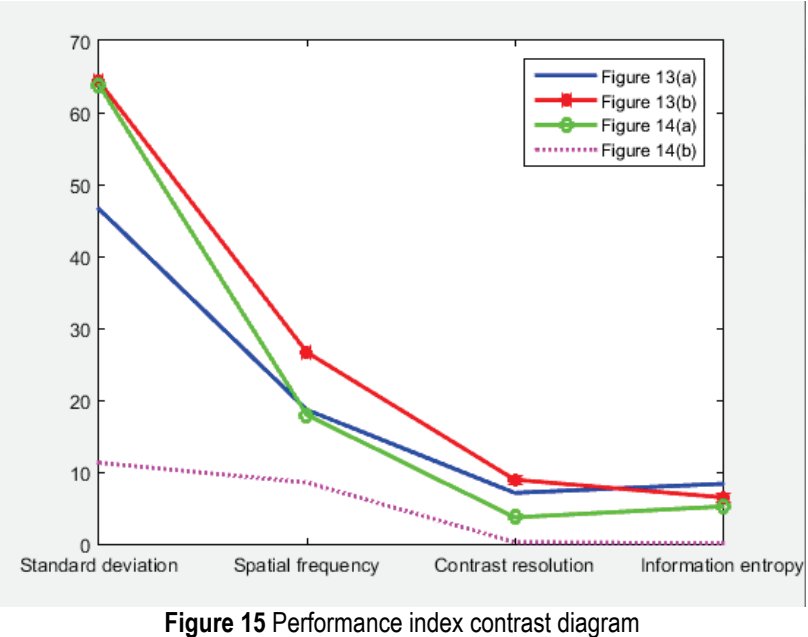

Table 2Performance index after wavelet transform fusion

\begin{tabular}{|c|c|c|c|c|}
\hline Parameter & $\begin{array}{c}\text { Standard } \\
\text { deviation }\end{array}$ & $\begin{array}{c}\text { Spatial } \\
\text { frequency }\end{array}$ & $\begin{array}{c}\text { Contrast } \\
\text { resolution }\end{array}$ & $\begin{array}{c}\text { Information } \\
\text { entropy }\end{array}$ \\
\hline Fig. 13a & 46.5806 & 18.6550 & 7.0963 & 8.3572 \\
\hline Fig. 13b & 64.3158 & 26.6502 & 8.9267 & 6.4740 \\
\hline Fig. 14a & 63.8202 & 17.9466 & 3.6867 & 5.2045 \\
\hline Fig. 14b & 11.2894 & 8.5402 & 0.2464 & 0.0757 \\
\hline
\end{tabular}

\section{CONCLUSION}

This paper proposes the target detection of visible and radar images based on wavelet transform of unmanned surface vehicles. The collected images are processed to extract the target features. The processed visible light and radar images are fused with wavelet transform strategy. The coefficients of the low frequency sub-band are processed by the average fusion strategy. The coefficients of the high frequency sub-band are processed using a strategy with a higher absolute value. The fused images are compared with four performance indexes: standard deviation, spatial frequency, contrast resolution and information entropy. The processed visible and radar images are fused to obtain the image whose effect is better, so that the target detection and recognition can be realized. The simulation results show that the processed image is better than the unprocessed image after the fusion. Moreover, the performance indexes of other fusion methods are obviously lower than the fusion methods adopted in this paper. In order to achieve the detection of targets, in the future simulation, the radar imaging (point track) will be used to detect the moving targets. The detection of target points can be obtained by fusion of visible image and radar image.

\section{Acknowledgements}

This article is funded by National Natural Science Fund (No. 61672304), Qiqihar City Science and Technology Project Fund (GYGG - 201626) and Graduate Innovation and Research Project Fund (YJSCX2017 ZD08).

\section{REFERENCES}

[1] Yousif, O. \& Ban, Y. (2017). A novel approach for objectbased change image generation using multitemporal highresolution SAR images. International Journal of Remote Sensing, 38(7), 1765-1787. https://doi.org/10.1080/01431161.2016.1217442

[2] Ham, B., Cho, M., \& Ponce, J. (2017). Robust guided image filtering using nonconvex potentials. IEEE Transactions on Pattern Analysis \& Machine Intelligence, 40(1), 192-207. https://doi.org/10.1109/TPAMI.2017.2669034

[3] Li, F. \& Lv, Z. (2017). Reliable vehicle type recognition based on information fusion in multiple sensor networks. Computer Networks, 117, 76-84. https://doi.org/10.1016/j.comnet.2017.02.013

[4] Li, C. B., Liu, Y. X., Zhang, H. et al. (2017). A false ship target discrimination algorithm in SAR image. Periodical of Ocean University of China, 47(2), 78-84. https://doi.org/10.16441/j.cnki.hdxb.20160279

[5] Karine, A., Toumi, A., Khenchaf, A. et al. (2017). Target recognition in radar images using weighted statistical dictionary-based sparse representation. IEEE Geoscience \& Remote Sensing Letters, PP(99), 1-5. https://doi.org/10.1109/LGRS.2017.2766225

[6] Chen, X. L., Guan, J., Dong, Y. L. et al. (2016). Sea clutter suppression and micromotion target detection in sparse domain. Chinese Journal of Electronics, 44(4), 860-867. https://doi.org/10.3969/j.issn.0372-2112.2016.04.015

[7] Tang, V. H., Bouzerdoum, A., \& Phung, S. L. (2018). Multipolarization through-wall radar imaging using lowrank and Jointly-sparse representations. IEEE Transactions on Image Processing, PP(99), 1-1. https://doi.org/10.1109/TIP.2017.2786462

[8] Ren, L., Tran, N., Foroughian, F. et al. (2018). Short-time state-space method for micro-Doppler identification of walking subject using UWB impulse Doppler radar. IEEE Transactions on Microwave Theory \& Techniques, PP(99), 1-14. https://doi.org/10.1109/TMTT.2018.2829523

[9] Zhang, S. H. (2017). A fast fusion algorithm of SAR and optical images based on improved $l_{1}$ norm and sparse representation. Sensor World, 23(4), 7-12. https://doi.org/10.16204/j.cnki.sw.2017.04.001

[10] Liu, J., Lei, Y. J., Xing, Y. Q., \& Lu, C. G. (2016). Fusion technique for SAR and gray visible image based on hidden Markov model in NSST domain. Control and Decision, 31(3), 453-457. https://doi.org/10.13195/j.kzyjc.2014.1932

[11] Rajah, P., Odindi, J., \& Mutanga, O. (2018). Feature level image fusion of optical imagery and synthetic aperture radar (SAR) for invasive alien plant species detection and mapping. Remote Sensing Applications Society \& Environment, 10, 198-208. https://doi.org/10.1016/j.rsase.2018.04.007

[12] Abergel, R., Denis, L., Ladjal, S. et al. (2018). Subpixellic methods for sidelobes suppression and strong targets extraction in single look complex SAR images. IEEE Journal of Selected Topics in Applied Earth Observations \& Remote Sensing, $P P(99), 1-18$. https://doi.org/10.1109/JSTARS.2018.2790987

[13] Machado, I., Toews, M., Luo, J. et al. (2018). Non-rigid registration of $3 \mathrm{D}$ ultrasound for neurosurgery using automatic feature detection and matching. International Journal of Computer Assisted Radiology \& Surgery, 13(2), 1-14. https://doi.org/10.1007/s11548-018-1786-7

[14] Hamdan, I., Bert, J., Cheze, C. L. R. et al. (2017). Fully automatic deformable registration of pre-treatment MRI/CT for image-guided prostate radiotherapy planning. Medical Physics, 44(12), 6447-6455. https://doi.org/10.1002/mp.12629

[15] Giordano, S., Mercier G., \& Rudant J. P. (2018). Unmixing polarimetric radar images based on land cover type identified by higher resolution optical data before target decomposition: Application to Forest and Bare Soil. IEEE Transactions on 
Geoscience \& Remote Sensing, PP(99), 1-13.

https://doi.org/10.1109/tgrs.2018.2827258

\section{Contact information:}

Dahui LI, PhD, Full Professor

(Corresponding author)

School of Computer and Control Engineering,

Qiqihar University,

Qiqihar, Heilongjiang, 161006, P. R. China

E-mail: 18645213579@163.com

\section{Qi FAN}

School of Computer and Control Engineering,

Qiqihar University,

Qiqihar, Heilongjiang, 161006, P. R. China

E-mail: 835491948@qq.com

\section{Jianzhao CUI}

School of Computer and Control Engineering,

Qiqihar University,

Qiqihar, Heilongjiang, 161006, P. R. China

E-mail: cuijz3x@163.com

Dehai HUANG, Lecturer

School of Computer and Control Engineering,

Qiqihar University,

Qiqihar, Heilongjiang, 161006, P. R. China

E-mail: 18845218090@163.com

Jinku HAN, Lecturer

School of Computer and Control Engineering,

Qiqihar University,

Qiqihar, Heilongjiang, 161006, P. R. China

E-mail: 948861806@qq.com 